\title{
The European Association of Urology Biochemical Recurrence Risk Groups Predict Findings on PSMA PET in Patients with Biochemically Recurrent Prostate Cancer After Radical Prostatectomy
}

\author{
Liang Dong ${ }^{1,2}$, Yun Su${ }^{3}$, Yinjie Zhu ${ }^{2}$, Mark C. Markowski ${ }^{4}$, Mei Xin ${ }^{5}$, Michael A. Gorin ${ }^{1}$, Baijun Dong ${ }^{2}$, Jiahua Pan ${ }^{2}$, \\ Martin G. Pomper ${ }^{6}$, Jianjun Liu ${ }^{5}$, Kenneth J. Pienta ${ }^{1}$, Wei Xue ${ }^{2}$, and Steven P. Rowe ${ }^{6}$ \\ ${ }^{1}$ James Buchanan Brady Urological Institute and Department of Urology, Johns Hopkins University School of Medicine, Baltimore, \\ Maryland; ${ }^{2}$ Department of Urology, Renji Hospital, Shanghai Jiao Tong University School of Medicine, Shanghai, China; ${ }^{3}$ Bloomberg \\ School of Public Health, Johns Hopkins University, Baltimore, Maryland; ${ }^{4}$ Department of Oncology, Sidney Kimmel Comprehensive \\ Cancer Center, Johns Hopkins University School of Medicine, Baltimore, Maryland; ${ }^{5}$ Department of Nuclear Medicine, Renji Hospital, \\ Shanghai Jiao Tong University School of Medicine, Shanghai, China; and ${ }^{6}$ Russell H. Morgan Department of Radiology and \\ Radiological Science, Johns Hopkins University School of Medicine, Baltimore, Maryland
}

Our purpose was to evaluate the association of a new biochemical recurrence $(\mathrm{BCR})$ risk stratification system with PSMA-targeted PET/CT findings. Methods: Two prospective studies that included patients with BCR were pooled. Findings on PSMA PET were catalogued. Patients were characterized according to the European Association of Urology BCR risk categories. Univariable and multivariable analyses were performed by logistic regression. Results: In total, 145 patients were included (45 low-risk and 100 high-risk). High-risk BCR patients had a higher positive rate than low-risk patients $(82.0 \%$ vs. $48.9 \% ; P<0.001$ ) and reached independent predictor status for positive PSMA PET/CT scan results on multivariable logistic regression (odds ratio, 6.73; 95\% Cl, 2.41-18.76; $P<0.001$ ). The area under the curve using the combination of BCR risk group and prostatespecific antigen was higher than that using prostate-specific antigen alone (0.834 vs. $0.759, P=0.015)$. Conclusion: The European Association of Urology BCR risk groups define the candidates who can most benefit from a PSMA PET/CT scan when BCR occurs.

Key Words: prostate cancer; BCR; prostate-specific membrane antigen; PET

J Nucl Med 2022; 63:248-252

DOI: 10.2967/jnumed.121.262411

\section{$\mathbf{P}$}

rostate cancer is the second most common cancer type and the fifth leading cause of cancer death in men worldwide (1). In patients who receive either radical prostatectomy (RP) or radiotherapy to treat their primary tumors, approximately $30 \%$ will develop biochemical recurrence (BCR) (2). Since, by definition, prostate cancer at this stage is invisible on conventional imaging, it is of importance to stratify BCR patients into different risk groups in order to give intensive treatment to patients with aggressive disease phenotypes.

Received Apr. 7, 2021; revision accepted May 20, 2021.

For correspondence or reprints, contact Steven P. Rowe (srowe8@jhmi. edu) or Wei Xue (uroxuewei@163.com).

Published online Jul. 29, 2021.

COPYRIGHT (C) 2022 by the Society of Nuclear Medicine and Molecular Imaging.
The European Association of Urology (EAU) BCR risk stratification system was proposed by the EAU prostate cancer guideline update, which defines low-risk BCR after RP as patients with a prostate-specific antigen (PSA) doubling time of more than 12 mo and a Gleason score of less than 8 ; high-risk BCR after RP is defined as patients with a PSA doubling time of no more than 12 mo or a Gleason score of at least 8 (3). Validation of this risk stratification system in 1,125 patients demonstrated that the 5-y metastatic progression-free and prostate cancer-specific mortalityfree survival rates were significantly higher among patients with low-risk BCR. Multivariable analysis confirmed the EAU risk stratification as an independent predictor of metastatic progression and prostate cancer-specific mortality (4).

With the recent advances in prostate-specific membrane antigen (PSMA) PET/CT, our current definition of BCR may soon be obsolete. We may need to begin rephrasing our clinical questions in the context of PSMA positivity. We previously reported that more than $60 \%$ of post-RP BCR patients had positive findings on PSMA PET/CT, and according to a metaanalysis, the positive predictive value of PSMA PET/CT was 0.99 based on a histopathologic gold standard (5-7).

The aim of the current study was to compare the detection rates and the localization of PSMA-avid lesions in low-risk versus highrisk BCR patients after RP and to evaluate the association of this new risk stratification system with PSMA PET/CT findings.

\section{MATERIALS AND METHODS}

\section{Patients}

We pooled cohorts of patients with BCR from 2 prospective studies at tertiary referral centers (Johns Hopkins Hospital and Renji Hospital). The inclusion criteria of the patients in each cohort, as well as technical details of the PSMA PET/CT scan (e.g., scanner, scan protocol, and scan interpretation) have been previously reported $(5,6)$. Risk stratification was performed as proposed by Van den Broeck et al. (3).

Pelvis-confined disease was defined by uptake of the radiotracer in the prostate bed, pelvic soft tissue, or pelvic lymph nodes. PSA doubling time was calculated as previously described (6), using the 3 most recent PSA values before PSMA PET/CT. If the slope of the 
linear regression was 0 (elevated but constant PSA) or negative (decreasing PSA after initial increase), the PSA doubling time was set as at least $12 \mathrm{mo}$.

\section{Statistical Analysis}

Logistic regression models were conducted for univariable and multivariable analyses, calculating odds ratios with $95 \%$ CIs to estimate the associations between BCR risk stratification and outcomes, adjusting for potential confounders. The predictive value of BCR risk stratification was assessed using the receiver-operating-characteristic curve and the area under the curve. Statistical testing was based on 2-sided tests at the 5\% level of significance. SAS software (version 9.4; SAS Institute) was used.

\section{RESULTS}

\section{Patients}

In total, 145 patients were enrolled; 94 were scanned with ${ }^{18}$ F-DCFPyL PET/CT (Johns Hopkins Hospital), and 51 were scanned with ${ }^{68}$ Ga-PSMA-11 PET/CT (Renji Hospital). Low-risk BCR was present in 45 patients, and high-risk BCR in 100. Table 1 summarizes the clinical and pathologic characteristics of these patients.

\section{Imaging Findings}

Of the 145 patients, $104(71.7 \%)$ had at least one PSMApositive lesion on the PSMA PET/CT scan. High-risk BCR patients had a significantly higher positive rate than the low-risk
BCR group ( $82.0 \%$ vs. $48.9 \%$; $P<0.001$; Fig. 1 A). On multivariable logistic regression analyses adjusted for age, PSA at the time of the scan, disease-free time, pathologic tumor stage (pT stage), and cohort (Johns Hopkins Hospital or Renji Hospital), the BCR risk group was an independent predictor for a positive PSMA PET/CT result (odds ratio, 6.73; 95\% CI, 2.41-18.76; $P<0.001$; Table 2). The median number of PSMA-positive lesions is 0 (interquartile range, $0-1$ ) for low-risk BCR and 1 (interquartile range, 1-3) for high-risk BCR. The multivariable linear regression model was used to estimate the associations between BCR risk group and lesion number. The model parameter $\beta$ is 0.85 , with statistical significance $(P=0.037)$.

In PSA subgroups, the positive rates of patients with low-risk BCR remained the same (40\%) in groups with a PSA of less than $0.5 \mathrm{ng} / \mathrm{mL}$ and with a PSA of $0.5-1.0 \mathrm{ng} / \mathrm{mL}$, whereas higher positive rates were observed with increasing PSA values in patients with high-risk BCR. Nearly $95 \%$ of patients with a PSA of more than $1.0 \mathrm{ng} / \mathrm{mL}$ in the high-risk group had detectable disease on PSMA PET/CT, whereas the positive rate was $66.7 \%$ for low-risk patients in the same PSA subgroup (Fig. 1B).

Of the 104 scan-positive patients, $56(53.8 \%)$ had pelvisconfined disease. The BCR risk group was not associated with pelvis-confined disease (Table 2; Fig. 1C). Receiver-operatingcharacteristic curves were generated to demonstrate the ability of the BCR risk group and PSA to predict positive PSMA PET/CT results. The areas under the curve using the BCR risk group or

TABLE 1

Demographics and Clinical Data for Study Cohort

\begin{tabular}{|c|c|c|c|}
\hline Parameter & BCR low risk & $\mathrm{BCR}$ high risk & $P$ \\
\hline Median age (y) & 71 (IQR, 65-76) & 69 (IQR, 63-73) & 0.426 \\
\hline Cohort & & & 0.288 \\
\hline Johns Hopkins Hospital & $32(71.1)$ & $62(62.0)$ & \\
\hline Renji Hospital & $13(28.9)$ & $38(38.0)$ & \\
\hline Median disease-free time (y) & $5.1(\mathrm{IQR}, 3-8)$ & $2.2(\mathrm{IQR}, 1.3-5.3)$ & 0.012 \\
\hline Adjuvant therapy & $44(97.8)$ & $86(86.0)$ & 0.242 \\
\hline Salvage therapy & $36(80.0)$ & $85(85.0)$ & 0.688 \\
\hline $\mathrm{PSA}(\mathrm{ng} / \mathrm{mL})$ at time of scan & & & 0.195 \\
\hline$<0.5$ & $20(44.4)$ & $31(31.0)$ & \\
\hline $0.5-1$ & $10(22.2)$ & $22(22.0)$ & \\
\hline$>1$ & $15(33.4)$ & $47(47.0)$ & \\
\hline PSADT $(\mathrm{mo})^{\star}$ & & & $<0.001$ \\
\hline$<12$ & $0(0.0)$ & $87(89.7)$ & \\
\hline$\geq 12$ & $45(100.0)$ & $10(10.3)$ & \\
\hline Gleason score & & & $<0.001$ \\
\hline$<8$ & $45(100.0)$ & $54(54.0)$ & \\
\hline$\geq 8$ & $0(0.0)$ & $46(46.0)$ & \\
\hline pT stage & & & 0.005 \\
\hline$<\mathrm{pT} 3$ & 33 (73.3) & $48(48.0)$ & \\
\hline$\geq \mathrm{pT} 3$ & $12(26.7)$ & $52(52.0)$ & \\
\hline
\end{tabular}

*PSA doubling time data of 3 Johns Hopkins Hospital patients are not available; however, all were high-risk based on Gleason scores. $\mathrm{BCR}=$ biochemical recurrence; IQR = interquartile range; PSADT = PSA doubling time.

Data are number followed by percentage in parentheses, unless specified otherwise. 

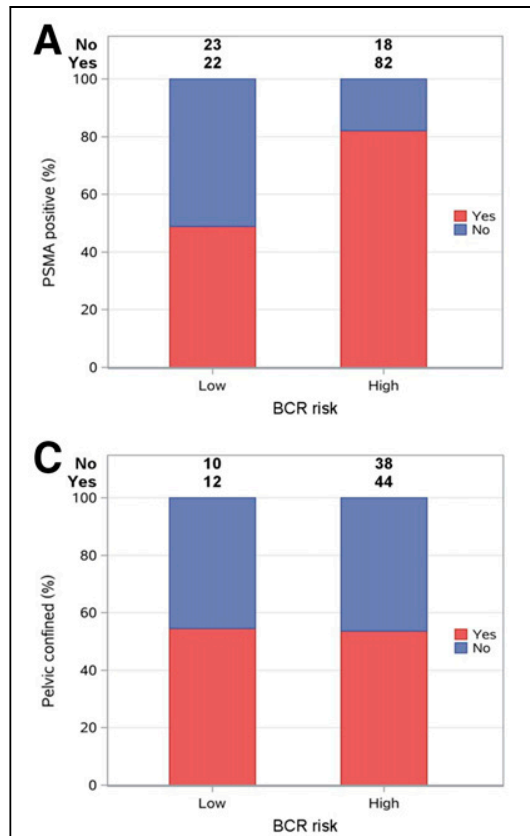

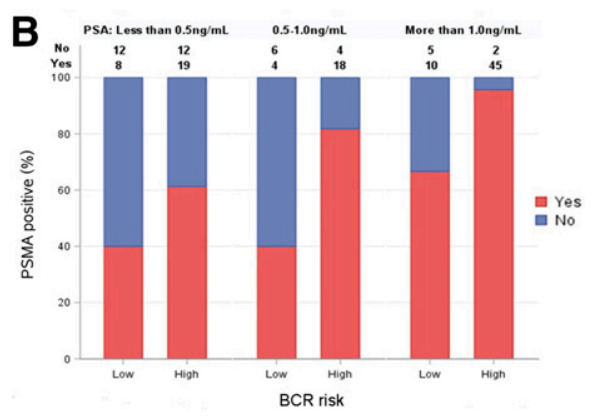

D

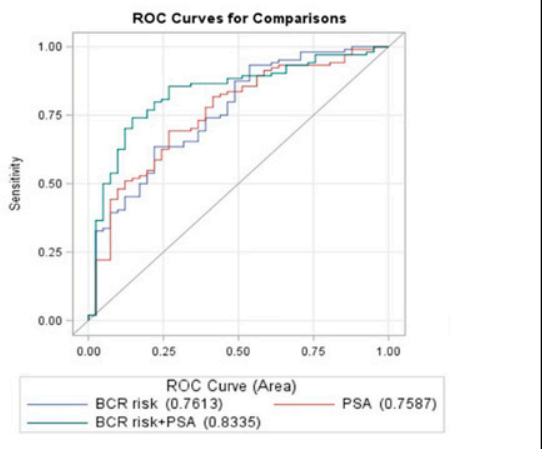

FIGURE 1. (A and B) Percentage of positive PSMA PET/CT scans among all patients (A) and among PSA subgroups (B). (C) Prevalence of pelvis-confined disease in each risk group. (D) Area under curve for detection of prostate cancer stratified by BCR risk group, PSA, and combination of $\mathrm{BCR}$ risk group and PSA. Each receiver-operating-characteristic multivariable analysis model also includes age, disease-free time, and pT stage. $\mathrm{LN}=$ lymph node; PB = prostate bed; ROC = receiver operating characteristic.

PSA alone were comparable ( 0.761 vs. $0.759, P=0.96$; Fig. $1 \mathrm{D})$, whereas the area under the curve using the combination of BCR risk group and PSA was higher than PSA alone (0.834 vs. 0.759 , $P=0.015$; Fig. 1D).

Of the 145 total patients, $68(46.9 \%)$ had recurrence or metastasis in lymph nodes, 28 (19.3\%) had bone metastasis, and 31 $(21.4 \%)$ had prostate bed recurrence. On multivariable logistic regression analyses, the BCR risk group was independently

associated with lymph node involvement on PSMA PET/CT in all patients, including those with negative scan results (odds ratio, 2.38; 95\% CI, 1.04-5.49; $P=0.041$; Table 2). However, in 104 patients with positive scan results, the BCR group was not associated with the location of PSMAavid lesions (Fig. 2; Table 2).

\section{DISCUSSION}

We demonstrated that patients with EAU high-risk BCR were more likely to have PSMA PET/CT-detectable disease, suggesting that tumor volume and distribution may help to explain the worse prognosis of those patients. Notably, even patients with low-risk BCR had relatively high detection rates on PSMA PET/CT, and the rates of extrapelvic disease on positive scans was similar between high- and lowrisk groups, suggesting that patients across the BCR spectrum may be good candidates for PSMA PET/CT imaging.

Previously, PSA has been reported as the strongest predictor of a positive PSMA PET/CT result (8). In this study, the added value of the EAU BCR risk groups has been demonstrated in a diverse population. It further stratifies the patients in each PSA subgroup, defining the patients who are most likely to have a positive PSMA PET/CT result. Use of EAU risk groups can serve as a simple and clinically applicable nomogram for predicting whether patients will have a positive scan result. The survival benefits from salvage pelvic radiation or focal treatment of oligometastases in different $\mathrm{BCR}$ risk groups in the context of PSMA PET/CT should be further explored.

The EAU BCR risk groups are associated with meaningful oncologic outcomes such as metastatic progression-free and

TABLE 2

Univariable and Multivariable Logistic Regression Models Stratified According to EAU BCR Risk Groups Predicting Positive Findings, Pelvis-Confined Disease, and Disease Location on PSMA PET/CT Imaging

\begin{tabular}{|c|c|c|c|c|c|c|}
\hline \multirow[b]{2}{*}{ Outcome } & \multicolumn{3}{|c|}{ Univariable analysis } & \multicolumn{3}{|c|}{ Multivariable analysis } \\
\hline & Odds ratio & $95 \% \mathrm{Cl}$ & $P$ & Odds ratio* & $95 \% \mathrm{Cl}$ & $P$ \\
\hline Positive PSMA PET/CT scan & 4.76 & $2.19-10.35$ & 0.000 & 6.73 & $2.41-18.76$ & 0.000 \\
\hline Pelvis-confined disease & 0.96 & $0.38-2.48$ & 0.941 & 1.31 & $0.43-3.96$ & 0.631 \\
\hline Lymph node involvement in all patients & 2.60 & $1.24-5.47$ & 0.012 & 2.38 & $1.04-5.49$ & 0.041 \\
\hline Bone metastasis in all patients & 3.24 & $1.05-9.96$ & 0.041 & 2.50 & $0.76-8.24$ & 0.133 \\
\hline Prostate bed recurrence in all patients & 1.71 & $0.68-4.33$ & 0.255 & 1.91 & $0.69-5.32$ & 0.216 \\
\hline Lymph node involvement in PSMA-positive patients & 1.10 & $0.41-2.94$ & 0.846 & 0.97 & $0.31-3.01$ & 0.960 \\
\hline Bone metastasis in PSMA-positive patients & 1.86 & $0.57-6.08$ & 0.303 & 1.44 & $0.38-5.48$ & 0.594 \\
\hline Prostate bed recurrence in PSMA-positive patients & 0.89 & $0.32-2.45$ & 0.816 & 0.93 & $0.29-3.02$ & 0.902 \\
\hline
\end{tabular}

${ }^{*}$ Adjusted for age, PSA, disease-free time, pT stage, and cohort. 


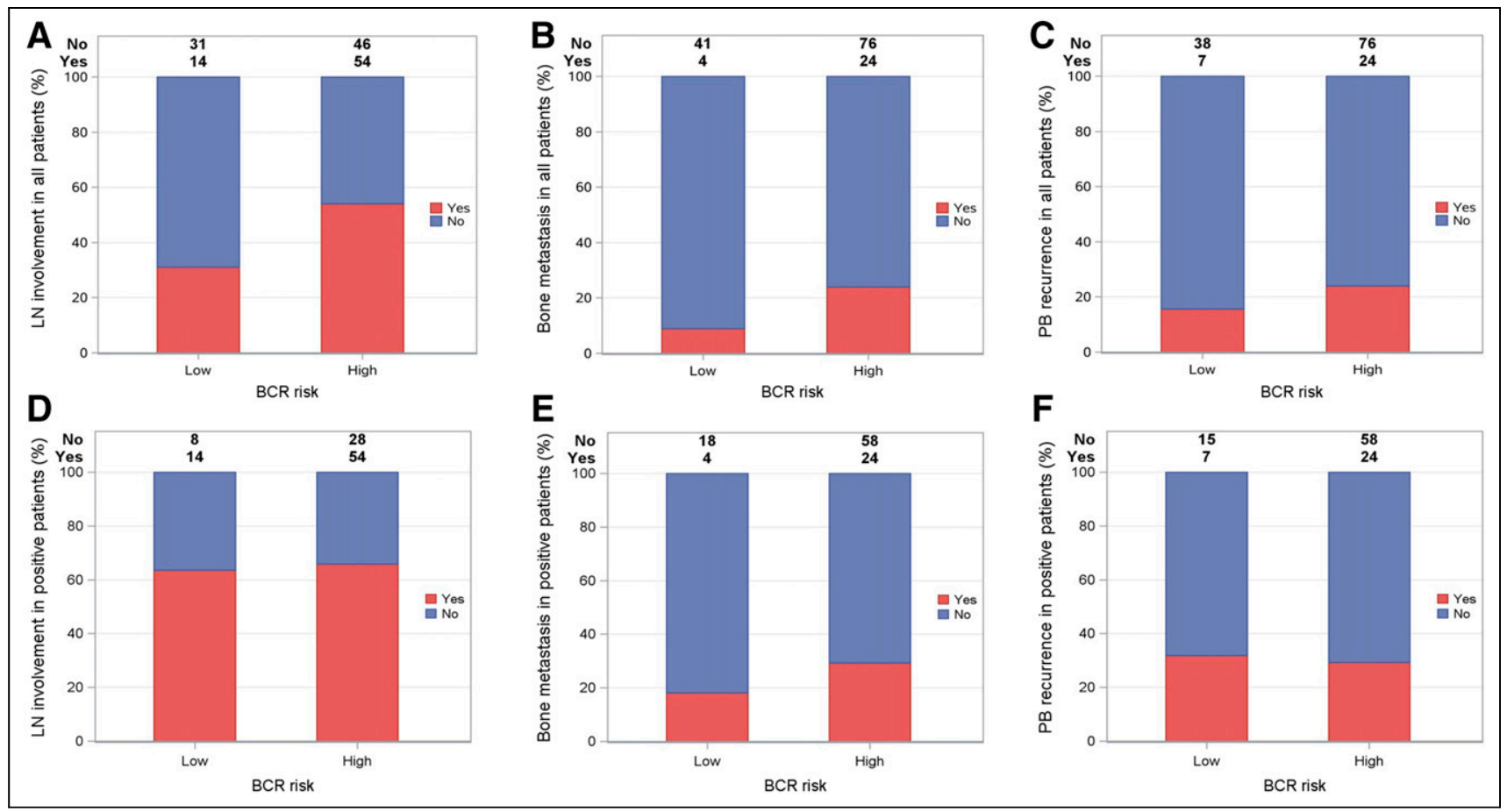

FIGURE 2. Percentage of all patients with lymph node involvement $(A)$, bone metastasis $(B)$, and prostate bed recurrence $(C)(n=145)$, and percentage of PSMA scan-positive patients with lymph node involvement (D), bone metastasis $(E)$, and prostate bed recurrence $(F)(n=104)$.

prostate cancer-specific mortality-free survival rates (4), suggesting that PSMA-targeted PET imaging will yield imaging biomarkers. Imaging specialists, urologists, and oncologists working with PSMA imaging should focus on the design of prospective trials that can discover and validate the prognostic significance of findings.

The limitations of this work include the relatively small number of cases, post hoc evaluation of prospectively acquired data, use of more than one PSMA-targeted radiotracer, and lack of central review or a specific read paradigm. Future work is needed to confirm these findings in multicenter, larger prospective cohorts.

\section{CONCLUSION}

The EAU BCR risk groups define the candidates who can most benefit from a PSMA PET/CT scan when BCR occurs.

\section{DISCLOSURE}

Martin Pomper is a coinventor on a U.S. patent covering ${ }^{18} \mathrm{~F}$-DCFPyL and as such is entitled to a portion of any licensing fees and royalties generated by this technology. This arrangement has been reviewed and approved by the Johns Hopkins University in accordance with its conflict-of-interest policies. Michael Gorin has served as a consultant to Progenics Pharmaceuticals, the licensee of ${ }^{18}$ F-DCFPyL. Steven Rowe is a consultant to Progenics Pharmaceuticals. Kenneth Pienta, Martin Pomper, Michael Gorin, and Steven Rowe have received research funding from Progenics Pharmaceuticals. Funding was received from Progenics Pharmaceuticals, the Prostate Cancer Foundation Young Investigator Award, the National Institutes of Health (grants CA134675, CA183031, CA184228, and EB024495), Program of Shanghai
Subject Chief Scientist (19XD1402300), and Program for Outstanding Medical Academic Leader (2019LJ11). No other potential conflict of interest relevant to this article was reported.

\section{ACKNOWLEDGMENTS}

We thank Meghan Pienta and Morgan D. Kuczler for their kind help in data collection.

\section{KEY POINTS}

QUESTION: Are the EAU BCR risk groups associated with findings on PSMA PET?

PERTINENT FINDINGS: In men with BCR after RP, the EAU high-risk group is more likely to have visible sites of recurrent disease on PSMA PET. However, low-risk and high-risk men have the same likelihood of having non-pelvis-confined disease.

IMPLICATIONS FOR PATIENT CARE: Risk stratification using the EAU BCR risk groups can help select men who are most likely to benefit from imaging with PSMA PET.

\section{REFERENCES}

1. Bray F, Ferlay J, Soerjomataram I, Siegel RL, Torre LA, Jemal A. Global cancer statistics 2018: GLOBOCAN estimates of incidence and mortality worldwide for 36 cancers in 185 countries. CA Cancer J Clin. 2018;68:394-424.

2. Han M, Partin AW, Zahurak M, Piantadosi S, Epstein JI, Walsh PC. Biochemical (prostate specific antigen) recurrence probability following radical prostatectomy for clinically localized prostate cancer. J Urol. 2003;169:517-523. 
3. Van den Broeck T, van den Bergh RC, Briers E, et al. Biochemical recurrence in prostate cancer: the European Association of Urology prostate cancer guidelines panel recommendations. Eur Urol Focus. 2020;6:231-234.

4. Tilki D, Preisser F, Graefen M, Huland H, Pompe RS. External validation of the European Association of Urology biochemical recurrence risk groups to predict metastasis and mortality after radical prostatectomy in a European cohort. Eur Urol. 2019;75:896-900.

5. Dong L, Zhu Y, Xin M, et al. Prospective evaluation of ${ }^{68}$ Ga-PSMA-11 PET/CT in Chinese men with biochemical recurrence after radical prostatectomy for prostate cancer: relationships between location of recurrence, time after prostatectomy, and serum PSA level. Med Oncol. 2020;37:89.
6. Markowski MC, Sedhom R, Fu W, et al. Prostate specific antigen and prostate specific antigen doubling time predict findings on ${ }^{18} \mathrm{~F}-\mathrm{DCFPyL}$ positron emission tomography/computerized tomography in patients with biochemically recurrent prostate cancer. $J$ Urol. 2020;204:496-502.

7. Hope TA, Goodman JZ, Allen IE, Calais J, Fendler WP, Carroll PR. Metaanalysis of ${ }^{68} \mathrm{Ga}$-PSMA-11 PET accuracy for the detection of prostate cancer validated by histopathology. J Nucl Med. 2019;60:786-793.

8. Perera M, Papa N, Roberts M, et al. Gallium-68 prostate-specific membrane antigen positron emission tomography in advanced prostate cancer: updated diagnostic utility, sensitivity, specificity, and distribution of prostate-specific membrane antigenavid lesions_-a systematic review and meta-analysis. Eur Urol. 2020;77:403-417.

\section{Erratum}

In the article "Comparative Prognostic and Diagnostic Value of Myocardial Blood Flow and Myocardial Flow Reserve After Cardiac Transplantation," by Miller et al. (J Nucl Med. 2020 Feb;61(2):249-255), Figures 1 and 4 contain errors.

In Figure 1, the AUC for corrected MFR should be 0.714 , as noted in the manuscript text as follows: "There were no significant differences in the ability of stress MBF (AUC, 0.713), MFR (AUC, 0.749), or corrected MFR (AUC, 0.714) to identify patients with significant CAV (Fig. 1)." In Figure 4, the labels for corrected and uncorrected MFR have been switched. The correct AUC for uncorrected MFR should be 0.748 and for correct MFR should be 0.724 . This is consistent with the current manuscript text as follows: "Uncorrected MFR showed improved discrimination for all-cause mortality compared with stress MBF (AUC, 0.748 vs. $0.639 ; P=0.048)$."

Due to these significant errors, we have re-reviewed the manuscript for any discrepancies between the manuscript text and tables/figures. Additionally, we have repeated all analyses to ensure accuracy. During this process, we have not identified any additional errors.

Corrected versions of Figures 1 and 4 appear below; the authors sincerely regret these errors.

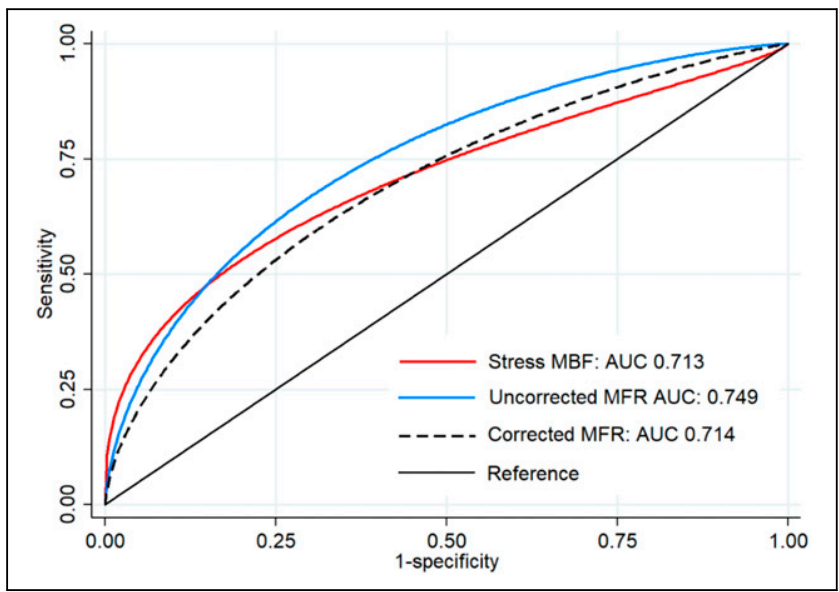

FIGURE 1.

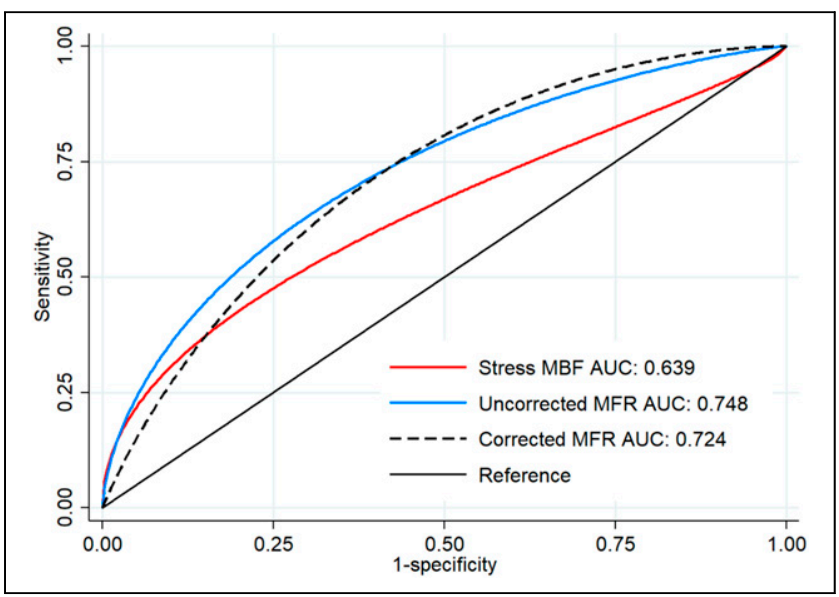

FIGURE 4. 